\title{
THE REGULATION OF POTENTIAL TRANSFORMERS AND THE MAGNETIZING CURRENT.
}

\author{
By M. G. Lloyd and P. G. Agnew.
}

The formulas now in general use for calculating the regulation of potential transformers involve the magnetizing current. Since the magnetizing current is flowing both at no load and at full load, and since regulation depends only on the difference in ratio between no load and full load, we should expect, a priori, the regulation to be independent of the magnetizing current. That it has no appreciable effect will be shown by developing the formula for regulation from the consideration of the vector diagram, from a treatment of the problem by the symbolic method, and by experimental results.

The regulation is defined as the change in secondary voltage between no load and full load (with constant primary voltage) expressed as a percentage of the secondary voltage on full load. In practice it is sometimes wrongly expressed in per cent of the noload value, but the error in so doing will seldom amount to more than one-tenth per cent.

The regulation may also be expressed as the change in ratio of terminal voltages $\left(\frac{\text { primary }}{\text { secondary }}\right)$ between no load and full load divided by the ratio at no load.

The difference between the induced and the terminal voltage of a transformer winding is due to its resistance and its leakage reactance. If these be known for both windings, the voltage drop is thereby determined, and the regulation may be calculated. Direct measurements of resistance and of impedance drop of voltage are easily made by well-known methods, and furnish the required data.

The relations involved may easily be seen by reference to figure $\mathrm{I}$, which is a vector diagram of the quantities concerned. Let $\Phi$ represent the magnetic flux, $E_{2}^{\prime}$ the voltage induced in the secondary winding, and $I_{2}$ the secondary current. The total current- 
turns $N_{1} I_{0}$ for exciting the core is made up of the secondary current-turns $N_{2} I_{2}$ and the primary $N_{1} I_{1}$.

The voltage $E_{1}$ applied to the primary is made up of three parts. One of these, $E_{1}^{\prime}$ opposite in phase to $E^{\prime}{ }_{2}$, balances the electromotive force induced by the core; a second part $I_{1} x_{1}$ in quadrature with the primary current balances the e. $\mathrm{m}$. f. due to leakage reactance; the third, equal to $I_{1} r_{1}$, the resistance drop of potential, is effective in sending current. The voltage induced in the secondary $E_{2}^{\prime}$ is similarly made up of three parts; $I_{2} r_{2}$ is the resistance drop in the winding, $I_{2} x_{2}$ the reactance drop, and $E_{2}$ the terminal voltage.

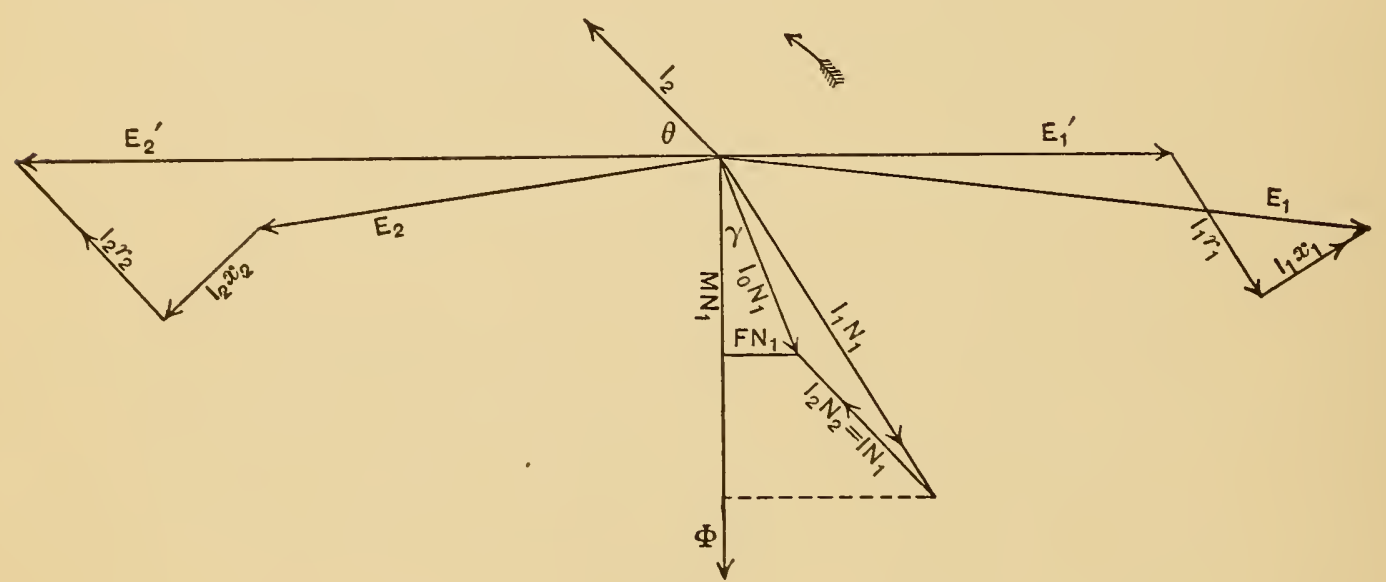

Fig. 1.

$E_{2}$ is less than $E_{2}^{\prime}$ except at no load, and is then equal to it; and since $E_{1}$ is always under practical working conditions greater than $E_{1}^{\prime}$, the ratio of primary to secondary voltage is greater than $\frac{E_{1}^{\prime}}{E^{\prime}}$ the ratio of turns.

In computing the regulation it is most convenient to consider the reactance and resistance of both windings lumped in one, say the primary. The equivalent total resistance of the primary, $R$, is found by multiplying the secondary resistance by the square of the ratio of turns and adding to the primary. The equivalent total reactance drop is found from the short-circuit test for impedance. Thus, if $e$ be the voltage necessary to send the rated full load current $I$ through the primary with secondary shortcircuited the total reactance drop is

$$
\sqrt{e^{2}-I^{2} R^{2}}=I X
$$


Now, for convenience, let us keep in mind that the primary current $I_{1}$ can be regarded as made up of two parts, $I$ in reversed phase with the secondary current and $I_{0}$, the exciting current. The primary resistance drop can also be divided into two parts, $I r_{1}$ and $I_{0} r_{1}$, of which the first is in reversed phase with the secondary resistance drop and may be lumped with it as $I R$. The difference between the terminal voltages is thus made up of $I_{0} r_{1}, I R$, $I X$, and the impedance drop of the exciting current, $I_{0} x_{1}$. Resolve each of these into two components, parallel and perpendicular to $\mathrm{E}_{2}^{\prime}$, for ease in combining. The parallel components are

$$
I_{0} r_{1} \sin \gamma+I R \cos \theta+I X \sin \theta+I_{0} x_{1} \cos \gamma
$$

The perpendicular components are

$$
I_{0} r_{1} \cos \gamma+I R \sin \theta-I X \cos \theta-I_{0} x_{1} \sin \gamma
$$

If we designate the two components of $\mathrm{I}_{0}$ as $M$ and $F$, and the ratio of turns by $n$, we have

$$
\begin{gathered}
\mathrm{E}_{1}^{2}=\left(n E_{2}+F r_{1}+I R \cos \theta+I X \sin \theta+M x_{1}\right)^{2} \\
+\left(M r_{1}+I R \sin \theta-I X \cos \theta-F x_{1}\right)^{2}
\end{gathered}
$$

Dividing by $E_{1}{ }^{2}$ and extracting the square root, we get

$$
\begin{aligned}
I=\frac{n E_{2}}{E_{1}} & +\frac{F r_{1}+I R \cos \theta+I X \sin \theta+M x_{1}}{E_{1}} \\
& +\frac{\mathrm{I}}{2 \mathrm{E}_{1}{ }^{2}}\left(M r_{1}+I R \sin \theta-I X \cos \theta-F x_{1}\right)^{2}
\end{aligned}
$$

with close approximation.

For no load, we have

$$
\mathrm{I}=\frac{n E_{2,0}}{E_{1}}+\frac{F r_{1}+M x_{1}}{E_{1}}+\frac{\mathrm{I}}{2 E_{1}^{2}}\left(M r_{1}-F x_{1}\right)^{2}
$$

Since the term in quadrature is always very small in comparison to the total voltage, we get by subtraction of this equation from the previous

$$
\frac{n E_{2,0}-n E_{2}}{E_{1}}=\frac{I R \cos \theta+I X \sin \theta}{E_{1}}+\frac{I}{2 E_{1}{ }^{2}}(I R \sin \theta-I X \cos \theta)^{2}
$$

Multiplying by $\operatorname{roo} \frac{E_{1}}{n E_{2}}$ we have the regulation expressed in per cent. 
IOO $\frac{E_{2,0}-E_{2}}{E_{2}}=\frac{\mathrm{IOO}}{n E_{2}}\left[I R \cos \theta+I X \sin \theta+\frac{\mathrm{I}}{2 E_{1}}(I R \sin \theta-I X \cos \theta)^{2}\right]$

where $n E_{2}$ may be substituted for $E_{1}$ without appreciable error. For non-inductive load this becomes ${ }^{1}$

$$
\text { гоo }\left[\frac{I R}{n E_{2}}+\frac{1}{2}\left(\frac{I X}{n E_{2}}\right)^{2}\right]
$$

For approximate work $E_{1}$ may be used in place of $n E_{2}$ and the reactance term neglected for noninductive load. If the reactance drop is not over 3 per cent, this term will affect the result by less than o.I per cent. If it is desired to get the result correct to o.or, it is necessary to use $n E_{2}$, which requires that the ratio of turns, $n$, be known, or that the ratio of voltages be measured and the approximate value of the above expression be used to determine the value of $n E_{2}$ to be used in a more exact computation. In some transformiers, $n$ is the same as the ratio of voltages given on the name plate, but in others, such as instrument transformers (where accurate values are most desired) the turns are slightly altered to give the nominal ratio at full load, or at half load.

For most practical purposes it is entirely sufficient to know the regulation to o.I per cent. It is customary in some quarters to compute the values of regulation to o.or per cent, whereas the conditions of use are not specified sufficiently closely to warrant it. Thus the cold resistance may be used in the computation, whereas the regulation after being loaded for some time might be the quantity desired. So that unless the temperature is specified, it is meaningless to consider regulation to o.or per cent.

It is evident from the above that although the exciting current affects the ratio slightly, it has an entirely inappreciable effect upon the regulation, or change of secondary voltage with load. The formulas published by some of the manufacturing companies are in error in this respect, since they contain the magnetizing current as one of the quantities affecting the regulation. These formulas are generally complicated to an unnecessary extent, also, by retaining the radical sign, instead of simplifying the expression as above. In the General Electric Review for December, 1908, one writer computes tables showing the alleged effect upon regula-

1 This formula is correctly given by LaCour in Arnold's "Wechselstromtechnik," Vol. I. 
tion of different values of magnetizing current, while as a matter of fact the magnetizing current has no such effect. Whatever objections there may be to the large magnetizing currents sometimes observed with cores of silicon steel, it does not consist in disordered regulation.

An example which recently came to our notice was in the case of an instrument transformer rated at 200 watts, $8660 / 100$ volts, 50 cycles, which required an exciting current of 0.737 ampere on the low-voltage side. The core loss was 35.8 watts; the impedance drop (primary side), 84.8 volts; primary resistance, I600 ohms; secondary resistance, $0.175 \mathrm{ohm}$. From these observed values the following are computed:

$$
\begin{aligned}
R & =2910 \\
I R & =67.2 \\
M & =0.00744 \\
F & =0.004 \mathrm{I} 3 \\
I X & =5 \mathrm{I} .7
\end{aligned}
$$

Substituting these values in the above equation for noninductive load, we get for the regulation, 0.78 per cent. Making the same calculation by the formula ${ }^{2}$ used in the tables mentioned above, we get for the regulation I.Io per cent. The difference is $0.3^{2}$, which represents the error introduced by using the wrong formula.

The regulation was experimentally determined by measuring the ratio at no load and at full load. A differential null method ${ }^{3}$ was used giving an accuracy of about one part in five thousand. The values of ratio found were $86.5^{2}$ at no load and 87.20 at full load, giving a value of regulation of $100 \frac{.68}{86.5^{2}}$ or 0.79 per cent.

In another transformer of the same type and capacity, but higher primary voltage, the exciting current measured on the secondary was 3.92 amperes, and it consequently presents an extreme case. The regulation as determined experimentally on non-inductive load was 0.29 per cent. The value according to our formula was 0.28 and by the other formula 2.56 , or nine times as large as the correct value.

${ }^{2}$ Substituting our notation, this formula is

$$
100 \sqrt{\left(\mathrm{I}+\frac{I R}{E_{1}}+\frac{M X}{E_{1}}\right)^{2}+\left(\frac{I X}{E_{1}}\right)^{2}}-100
$$

${ }^{3}$ For a description of this method, see Agnew and Fitch, this Bulletin, p. 28 I. 
In power transformers the exciting current is relatively smaller and the resistance drop relatively larger, and the errors are consequently much less in magnitude. It is probably due to this fact that incorrect formulas have been used for so long without exciting suspicion.

In order to test the effect of varying magnetizing current upon the same transformer, some measurements were made at different voltages upon a 600-watt, 60-cycle, I20/ I 20-volt transformer. The results are given in Table $I$. At 80 volts the exciting current was 0.27 and the ratios at no load and full load were 1.0007 and r.0348. At I 50 volts the exciting current was $\mathrm{I} .33$ and the ratios r.oor 8 and r.org9. Full load in each case consisted of lamps

TABLE I.

\begin{tabular}{c|c|c|c|c}
\hline Primary volts & Secondary amperes & Exciting amperes & Ratio & Regulation drop \\
\cline { 2 - 4 } 130 & 5.0 & & 1.0220 & 2.70 \\
130 & 0.0 & 0.752 & 1.0012 & \\
140 & 5.0 & & 1.0207 & 2.70 \\
140 & 0.0 & 0.973 & 1.0014 & \\
120 & 5.0 & & 1.0236 & 2.71 \\
120 & 0.0 & 0.580 & 1.0010 & \\
100 & 5.0 & & 1.0281 & 2.73 \\
100 & 0.0 & 0.354 & 1.0008 & \\
80 & 5.0 & & 1.0348 & 2.73 \\
80 & 0.0 & 0.27 & 1.0007 & \\
150 & 5.0 & & 1.0199 & 2.72 \\
150 & 0.0 & 1.328 & 1.0018 & \\
\hline
\end{tabular}

carrying five amperes. It is at once evident that exciting current affects ratio, since the latter is not constant for no load. To determine whether the exciting current affects the regulation, we must determine whether the difference in actual drop varies in the two cases. The difference in ratio between no load and full load gives the drop in terms of the voltage; multiplying this by the voltage gives the actual drop. At 80 volts this amounts to $.034 \mathrm{I} \times 80=2.728$. At 150 volts it is $.018 \mathrm{I} \times 150=2.7 \mathrm{I} 5$, an 
agreement within one-half of one per cent of the value of the drop, or .or per cent of the total voltage. This makes it evident that if the exciting current could be changed without altering the voltage (say by changing the core) the ratio of terminal voltages would be changed, but the regulation would not be changed, since the changes in ratio at no load and full load would be of equal magnitude.

The above difference of or per cent may well be attributed to slight changes in resistance from heating during the experiment, although the changes were made as rapidly as possible from one condition to the other. Readings were also taken at other intermediate voltages, in irregular order, and all gave the same result within .or per cent of the mean.

The derivation of the same formula for regulation by the use of complex quantities, in place of the geometrical or vector method, follows:

$r_{1}=$ primary resistance; $r_{2}=$ secondary resistance. $R=r_{1}+n^{2} r_{2}$. $x_{1}=$ primary reactance; $x_{2}=$ secondary reactance. $X=$ total reactance referred to primary.

$F+j M=$ exciting current.

$P+j W=$ load current (on primary side).

$n=$ ratio of turns.

$E_{1}=$ terminal primary voltage.

$E+j Q=E_{2}=$ terminal secondary voltage.

$E_{1}-\left(r_{1}+j x_{1}\right)(F+j M+P+j W)$

$$
\begin{aligned}
& =n(E+j Q)+n\left(r_{2}+j x_{2}\right)(n P+n j W) \\
E_{1}-r_{1}(F+P)+x_{1}(M+W) & =n E+n^{2}\left(r_{2} P-x_{2} W\right)
\end{aligned}
$$

or

$$
\begin{aligned}
E_{1}-r_{1} F-R P+x_{1} M+X W & =n E \\
-r_{1}(M+W)-x_{1}(F+P) & =n Q+n^{2} r_{2} W+n^{2} x_{2} P
\end{aligned}
$$

or

$$
-r_{1} M-R W-x_{1} F-X P=n Q
$$

From (I) we have for the drop in voltage in phase with $E_{1}$

$$
E_{1}-n E=r_{1} F+R P-x_{1} M-X W
$$


For no load

$$
E_{1}-n E_{0}=r_{1} F-x_{1} M
$$

From (2) we have for the drop in voltage in quadrature

For no load

$$
-n Q=r_{1} M+R W+x_{1} F+X P
$$

$$
-n Q_{0}=r_{1} M+x_{1} F
$$

For regulation it is sufficient practically to take differences of real and imaginary parts before combining. Then for the regulation drop in phase and in quadrature, we have

$$
\begin{aligned}
& n\left(E_{0}-E\right)=R P-X W \\
& n\left(Q_{0}-Q\right)=R W+X P
\end{aligned}
$$

and the regulation in per cent is

$$
\begin{aligned}
\frac{\mathrm{IO0} \sqrt{\left(n E_{2}+R P-X W\right)^{2}+(R W+X P)^{2}}}{n E_{2}}-100 \\
=100 \sqrt{\left(1+\frac{R P}{n E_{2}}-\frac{X W}{n E_{2}}\right)^{2}+\left(\frac{R W+X P}{n E_{2}}\right)^{2}}-100 \\
\quad=100\left[\frac{R P-X W}{n E_{2}}+\frac{1}{2}\left(\frac{R W+X P}{n E_{2}}\right)^{2}\right]
\end{aligned}
$$

For non-inductive load, $W=o$, and this reduces to

$$
\operatorname{IOO}\left[\frac{R P}{n E_{2}}+\frac{\mathrm{I}}{2}\left(\frac{X P}{n E_{2}}\right)^{2}\right]
$$

It should be borne in mind that the proper algebraic sign must accompany numerical values of $M$ and $W$ when these are introduced into the formula. These will be positive for leading and negative for lagging values. Consequently $M$ is always negative and $W$ usually so.

WASHINGTON, June 2I, I909. 